\title{
PRMT5 regulates Golgi apparatus structure through methylation of the golgin GM130
}

\author{
Zhongwei Zhou ${ }^{1, *}$, Xiaotian Sun ${ }^{1,2,{ }^{*}}$, Zhenhua Zou ${ }^{1,2}$, Litao Sun $^{3}$, Tao Zhang ${ }^{1,2}$, Shaoshi Guo ${ }^{1,2}$, Ya Wen ${ }^{1}$, \\ Lin Liu ${ }^{3}$, Yi Wang ${ }^{4}$, Jun Qin ${ }^{4}$, Lei Li $^{5}$, Weimin Gong ${ }^{3}$, Shilai Bao ${ }^{1}$ \\ ${ }^{I}$ Key Laboratory of Molecular and Developmental Biology, Institute of Genetics and Developmental Biology (IGDB), Chinese \\ Academy of Sciences, Beijing 100101, China; ${ }^{2}$ Graduate School of the Chinese Academy of Sciences, Beijing 100039, China; ${ }^{3} I n-$ \\ stitute of Biophysics, Chinese Academy of Sciences, Beijing 100101, China; ${ }^{4}$ Department of Biochemistry and Molecular Biology, \\ Baylor College of Medicine, Houston, Texas 77030, USA; ${ }^{5}$ Department of Experimental Radiation Oncology, MD Anderson Can- \\ cer Center, Houston, Texas 77030, USA
}

Maintenance of the Golgi apparatus (GA) structure and function depends on Golgi matrix proteins. The posttranslational modification of Golgi proteins such as phosphorylation of members of the golgin and GRASP families is important for determining Golgi architecture. Some Golgi proteins including golgin-84 are also known to be methylated, but the function of golgin methylation remains unclear. Here, we show that the protein arginine methyltransferase 5 (PRMT5) localizes to the GA and forms complexes with several components involved in GA ribbon formation and vesicle tethering. PRMT5 interacts with the golgin GM130, and depletion of PRMT5 causes defects in Golgi ribbon formation. Furthermore, PRMT5 methylates N-terminal arginines in GM130, and such arginine methylation appears critical for GA ribbon formation. Our findings reveal a molecular mechanism by which PRMT5-dependent arginine methylation of GM130 controls the maintenance of GA architecture.

Keywords: Arginine methylation; GM130; Golgi structure; PRMT5

Cell Research (2010) 20:1023-1033. doi:10.1038/cr.2010.56; published online 27 April 2010

\section{Introduction}

The Golgi apparatus (GA) plays a central role in the posttranslational modification, sorting, and transportation of proteins. In mammalian cells, the GA is a dynamic structure, which is broken down into small vesicles and tubules during mitosis and is reassembled from these fragments into stacks during telophase, and these stacks are linked to form a ribbon during interphase $[1,2]$. GA membrane fusion and fragmentation are closely related to two sets of proteins, a family of coiled-coil proteins called golgins and Golgi reassembly stacking proteins (GRASPs) [3, 4]. The best characterized golgins are p115 and GM130, which are thought to form a tethering

\footnotetext{
*These two authors contributed equally to this work. Correspondence: Shilai Bao

Tel: +86-10-64889350; Fax: +86-10-64854896

E-mail: slbao@genetics.ac.cn

Received 4 January 2010; revised 16 March 2010; accepted 24 March 2010; published online 27 April 2010
}

complex that recruits COPII vesicles to fuse with cisGolgi membrane $[5,6]$. GRASP65, a protein involved in Golgi stack formation, binds directly to GM130 to target this complex to the cis-Golgi [7]. It has been proposed that $\mathrm{p} 115$, GM130 and Giantin form a tripartite tether involved in the fusion of recycling COPI vesicles $[8,9]$, and that the GM130-GRASP65 complex is required for lateral cisternal fusion to form the Golgi ribbon [10]. Studies on a temperature-sensitive cell line revealed that detectable levels of GM130 was not required for normal GA structure in mammalian cells at the permissive temperature, suggesting that membrane tethering may be supported by redundant functions [11].

At the onset of mitosis in mammalian cells, the GA undergoes extensive fragmentation. This process is linked to protein phosphorylation. The p115-GM130 tethering complex is disrupted by CDK1-mediated phosphorylation of GM130 on serine 25 during mitosis. This contributes to the attenuation of vesicle transport and perturbation of Golgi structure [6, 12]. Phosphorylation of GRASP65 and GRASP55 is thought to be critical for 
Golgi membrane fragmentation when cells enter mitosis [13]. Golgin-84 is a phosphoprotein in metaphase, but the function of its phosphorylation in regulation of GA structure remains unknown [14].

In addition to phosphorylation, other posttranslational modifications such as acetylation and ubiquitination are important in regulation of GA structure and transport [15, 16]. It has also been reported that golgin-84, GRASP55 and other GA-localized proteins can be methylated on arginines [17]. However, the biological function of arginine methylation of GA-localized proteins has not been elucidated.

Protein arginine methyltransferase PRMT5, also called JBP1 [18] or Skb1 [19], is involved in RNA splicing [20], histone modification regulation of gene transcription [21$24]$, cell proliferation $[25,26]$, and differentiation [2729]. The nuclear localized function of PRMT5 regulating H4R3 methylation and RNA splicing has been studied intensively. Interestingly, fission yeast Skb1 localizes to both nucleus and cytoplasm [30], and mammalian PRMT5 primarily localizes to the cytoplasm in somatic cells such as 293T, COS-1, Chang liver, U2OS, and normal B-cell [31-33], as well as in cord blood progenitors and mouse primordial germ cells after embryonic day $11.5[23,27]$. PRMT5 is highly expressed in both the nucleus and cytoplasm in transformed mantle cell lymphoma cell lines and is enriched in the nucleus in patient samples [32]. It has been reported that PRMT5 translocation from cytoplasm to nucleus is dependent on coexpression of AJUBA and SNAIL in U2OS cells [33], whereas, in the case of differentiation of mouse primordial germ cells and human fetal gonocytes, its relocation from the nucleus to cytoplasm may be due to loss of its binding partner Blimp1 [27, 34, 35]. It has been proposed that PRMT5 relocates from nucleus to cytoplasm, where it may play a role in regulating pluripotency [35, 36]. However, little is known of the function of PRMT5 that retains in the cytoplasm. In this study, we investigated the cytoplasmic function of PRMT5. We show that PRMT5 interacts with GM130, localizes to the GA and regulates Golgi ribbon formation through methylation of GM130.

\section{Results}

\section{Identification of PRMT5 complexes}

To gain further insight into the cytoplasmic functions of PRMT5, we undertook biochemical purification of PRMT5 protein complexes from the cytosol of HeLa cells and identified PRMT5-associated proteins by mass spectrometry. PRMT5 was copurified with an additional 13 polypeptides (Figure 1A and Supplementary infor- mation, Table S1), including MEP50, a component of the SMN complex that has been shown to interact with PRMT5 [37], the golgin GM130 [5], the AAA ATPase NSF [38], and the GTPase-activating protein IQGAP1 [39]. These proteins are important for GA structure, vesicle transport, and cellular mobility, suggesting that PRMT5 may function in these processes. GM130 is of particular interest based on its function in the Golgi structure $[5-7,10,40]$. To further verify whether GM130 is associated with PRMT5, we performed reciprocal coimmunoprecipitation with anti-GM130 and antiPRMT5 antibodies. As shown in Figure 1B, a reciprocal interaction was confirmed between GM130 and PRMT5, consistent with our mass spectrometry result. PRMT5 and GM130 were not associated with golgin-84 (Figure 1B).

To further investigate the interaction between PRMT5 and GM130, in vitro protein binding analysis were performed. Flag-tagged PRMT5 was purified from the Chinese hamster ovarian $(\mathrm{CHO})$ cells stably expressing this construct and incubated with recombinant GST-tagged GM130 N-terminus (N, 1-500 aa), GM130 C-terminus (C, 497-990 aa), or GST. Flag-PRMT5 could pull down GST-GM130N, but not GST-GM130C or GST, and PRMT5 binding to GM130 was further mapped to the $\mathrm{N}$-terminal 73 residues using a shorter $\mathrm{N}$-terminal fusion (1-73 aa) (GM130N73) (Figure 1C). Conversely, recombinant GST-GM130N, GST-GM130C, or GST was incubated with $\mathrm{CHO}$ whole cell lysates containing FlagPRMT5 and then precipitated with GST beads. FlagPRMT5 was only associated with GST-GM130N, but not with GST-GM130C or GST (Figure 1D). These results indicate that PRMT5 physically interacts with the Nterminus of GM130.

\section{PRMT5 localizes to $G A$}

To reveal the cellular localization of PRMT5 in mammalian cells, we performed immunofluorescence microscopy studies with PRMT5 antibody. We found that the PRMT5 localized predominantly to the perinuclear region within the cytoplasm of U2OS cells, suggesting a possible GA localization. We then used various GA markers P230, ManII, GM130, Ginatin, NSF, and GRASP55 to determine whether PRMT5 localizes to the GA (Figure 2A and Supplementary information, Figure S1). PRMT5 exhibited significant overlap with these Golgi markers, but no colocalization signal was found between PRMT5 and the ER protein Calnexin (Supplementary information, Figure S1). The colocalization of PRMT5 with GA markers was also observed in other cell lines such as MCF7, HeLa, and HCT116 (data not shown).

To further confirm that PRMT5 is a GA-localized 
A

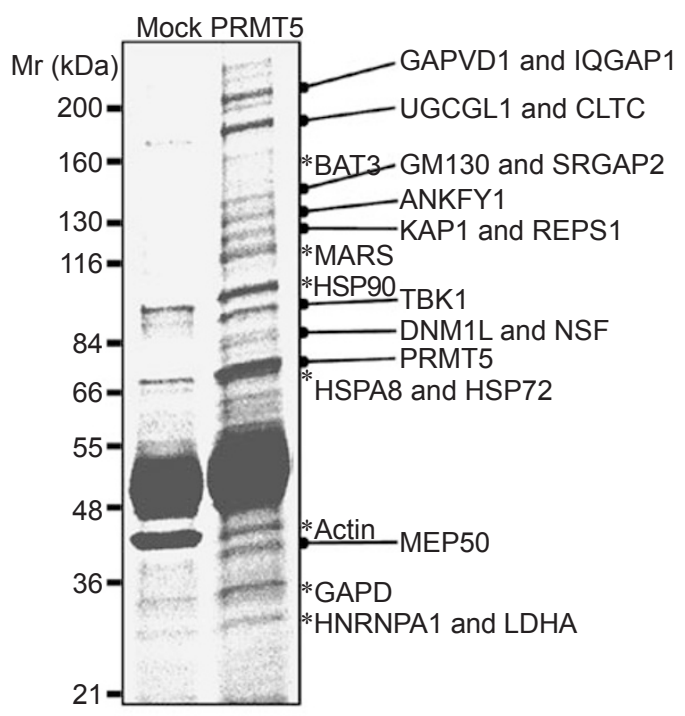

B

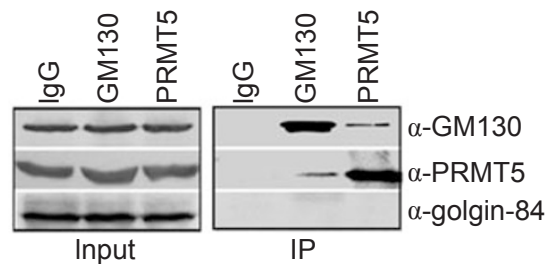

C

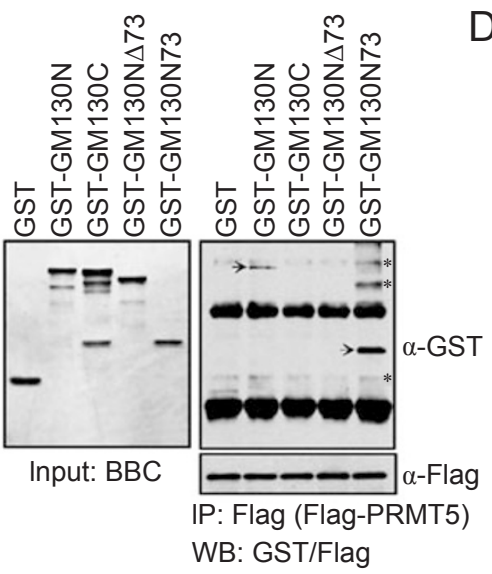

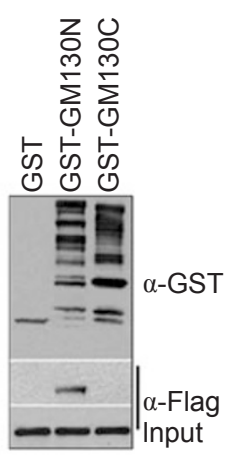

Pull down: GST

WB: GST/Flag

Figure 1 PRMT5 forms complexes with Golgi proteins. (A) S100 cytoplasm fraction from HeLa cells was immunoprecipitated with PRMT5 antibody. Mock purification eluted only the IgG. The PRMT5 immunoprecipitate was separated on a 4-20\% gradient SDS-PAGE and stained with Coomassie Blue. Protein bands were identified by mass spectrometry and indicated at right. Heat-shock proteins and actin (asterisks) are most likely nonspecifically associated components, as they are also present in IgG control elute. (B) Reciprocal immunoprecipitation (IP) of PRMT5 and GM130 with anti-PRMT5 and GM130 antibodies, donkey IgG as a negative control. The immunoprecipitates were separated on SDS-PAGE, transferred to membrane, and blotted with antibodies as indicated. Input is $10 \%$ amount of protein used in copurification assays. (C, D) Analysis of PRMT5 interaction with GM130 in vitro. Purified recombinants $(2 \mu \mathrm{g})$ of GST-GM130N, GST-GM130C, GST-GM130N $\Delta 73$, GSTGM130N73, or GST were mixed with $1 \mathrm{mg}$ clarified whole cell extracts of CHO cells with stable expression of Flag-PRMT5 and incubated at $4{ }^{\circ} \mathrm{C}$ for $12-14 \mathrm{~h}$. Mixtures were immunoprecipitated either with Flag antibodies (C) or with GST antibodies (D). Western blot analysis was performed with GST and Flag antibodies. Input (C, left panel) was recombinant proteins in SDSPAGE gel stained with Coomassie blue (BBC, upper) and $100 \mu \mathrm{g}$ extracts of $\mathrm{CHO}$ with Flag-PRMT5 immunoblotted with Flag antibodies (lower panel). Arrow points to specific associated protein band; nonspecific binding protein is indicated by asterisk.

protein, purified GA membranes were isolated from rat liver cells and analyzed by western blotting. PRMT5 and the Golgi proteins, GM130 and p115, were present in the GA fraction, but not ER, early endosome, mitochondrion, or plasma membrane proteins (Bip, EEA1, Bcl2, and Annexin II) (Figure 2B). These findings indicate that PRMT5 is indeed present in the Golgi apparatus.

\section{PRMT5 knockdown disrupts Golgi structure}

To examine whether PRMT5 regulates GA structure, we used a short hairpin loop-based shRNA vector [41] to knockdown PRMT5 expression, which reduces the protein level by more than $90 \%$ (Figure $3 \mathrm{~A}$ and Supplementary information, Figure S2). Under this condition, compared to cells transfected with a control shRNA, immunostaining signals of GM130, p230, NSF, ManII, and GRASP55 were significantly changed in PRMT5 knockdown cells (Figure 3B and Supplementary information,
Figure S3). Under microscopy, we found three types of GA structures that we classified as compact ribbon $(\mathrm{CR})$, ribbon $(\mathrm{R})$, and fragmented $(\mathrm{F})$ in the control and PRMT5 knockdown cells during interphase. Quantitation revealed that the ratio of CR:R:F was about 48:14:38 in the PRMT5-depleted cells, in contrast, the ratio of CR:R:F was 32:49:19 in the control cells (Figure 3C). Thus, disruption of PRMT5 resulted in increased Golgi ribbon fragmentation, suggesting that PRMT5 might influence GA structure by affecting tethering or fusion of Golgi membranes. To examine the effect of PRMT5 depletion on Golgi ultrastructure, electron microscopy was performed. This revealed that the Golgi stacks were either fragmented into clusters of vesicular elements or present as small isolated stacks in PRMT5-depleted cells (Figure 3D and Supplementary information, Figure S4). Cotransfection of a GFP-PRMT5-sm expression vector, which produced an shRNA-resistant wild-type PRMT5 

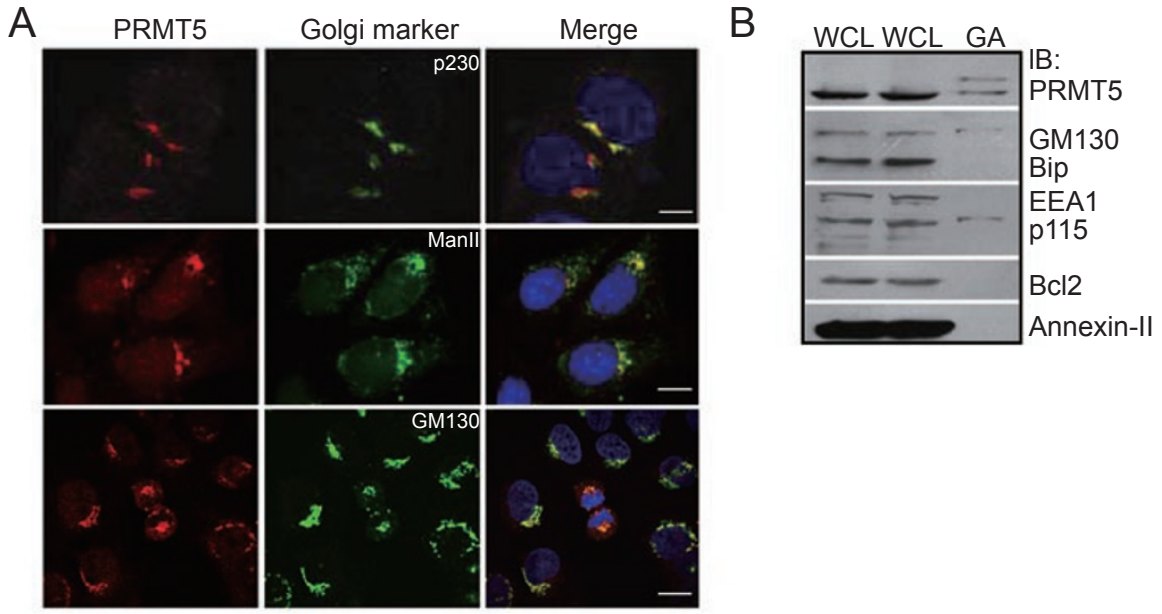

Figure 2 PRMT5 localizes to GA. (A) U2OS cells were costained with rabbit antibody to PRMT5 and the staining was visualized by a Cy3-conjugated secondary antibody (red), and with mouse antibodies to Golgi markers P230, Manll, and GM130, and the staining was visualized by a FITC-conjugated secondary antibody. Fluorescence images were acquired by confocal microscopy. Scale bars, $10 \mu \mathrm{m}$. (B) $100 \mu \mathrm{g}$ of proteins from rat liver whole cell lysate (WCL) and $10 \mu \mathrm{g}$ of purified rat liver Golgi apparatus (GA) proteins were loaded and immunoblotted with indicated antibodies.

protein (Figure 3E), was able to restore the GA ribbon structure as indicated by GRASP55 immunostaining in cells devoid of endogenous PRMT5 (Figure 3F and 3G). Collectively, these results provide strong evidence that PRMT5 is important for the maintenance of GA structure.

Next, we asked whether PRMT5 methyltransferase activity is required for its role in the maintenance of GA structure. We constructed a GFP-tagged PRMT5md vector, in which two amino acid substitutions were introduced (G367A/R368A) to inactivate PRMT5 methyltransferase activity [25]. Transfection of this vector did not induce GA ribbon fragmentation (data not shown), suggesting that the PRMT5 methyltransferase-dead mutant may not function as dominant negative. To eliminate the potential interference of the endogenous wild-type PRMT5, we constructed a GFP-PRMT5-dm vector, in which the shRNA-resistant GFP-PRMT5-sm vector was further mutated to introduce the G367A/R368A mutation to encode an shRNA-resistant and methyltransferasedead protein. GA ribbon structure could not be restored by GFP-PRMT5-dm after endogenous PRMT5 was depleted by shRNA (Figure 3F and 3G). Thus, PRMT5 methyltransferase activity is indispensable for the maintenance of GA structure.

\section{PRMT5 methylates N-terminal arginines of GM130 both in vitro and in vivo}

We next examined whether PRMT5 can methylate arginine residues of components of PRMT5 complexes.
PRMT5 complexes were isolated from HeLa cells, and incorporation of a radioactive methyl group from tritiumlabeled $S$-Adenosine Methionine (SAM), indicating methylation, was monitored by autoradiography. Several components were obviously methylated by PRMT5, including a major labeled protein migrating at $130 \mathrm{kDa}$ (Figure 4A, lanes 1 and 3). Mass spectrometry identified this protein as GM130 (data not shown). Moreover, when Flag-tagged full-length GM130 isolated from $\mathrm{CHO}$ cells was added to the PRMT5 complexes (Figure 4A, lane 4), the methylation signal at the position corresponding to GM130 was significantly increased (Figure 4A, lane 2), consistent with GM130 being a substrate of PRMT5. To further test this possibility, we generated recombinant PRMT5 and GM130 proteins in E. coli and performed an in vitro methylation assay. As shown in Figure 4B, a $31 \mathrm{kDa}$ GST fusion protein derived from the N-terminus of GM130 (GST-GM130N) was methylated by PRMT5, while no methylation was observed with GST or GM130 C-terminal GST fusion protein (GST-GM130C), implying that PRMT5 methylates arginine residue(s) in the N-terminus of GM130. Subsequent mass spectrometry analysis of the $31 \mathrm{kDa}$ methylated polypeptide identified Arg 6 and Arg18 or Arg23 as the residues methylated by PRMT5 (Supplementary information, Figure S5). We note that a full-length GST-GM130N could not be methylated by GST-PRMT5 (Figure 4B), which may be due to the incorrect folding of GST-GM130N purified from bacteria, as GST-GM130N contains a long coil-coiled domain at $\mathrm{N}$-terminus. 
A

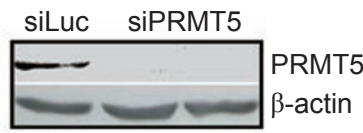

B

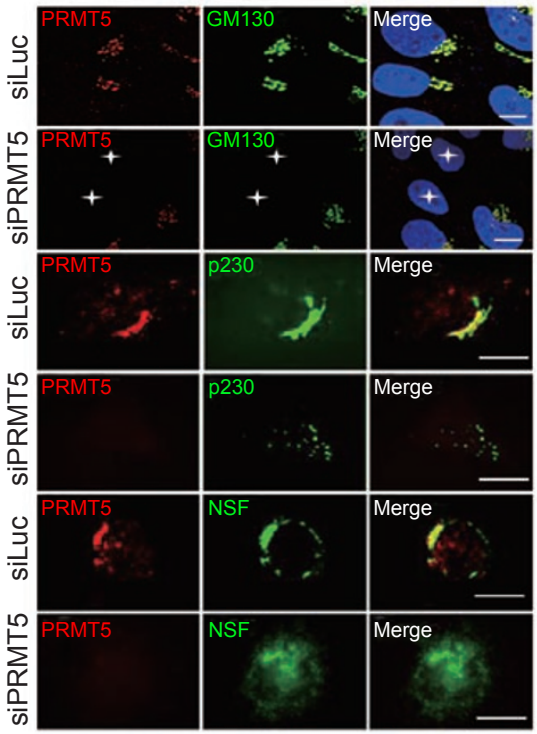

C

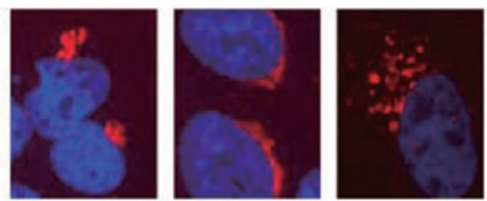

$50+\quad \square$ siluc

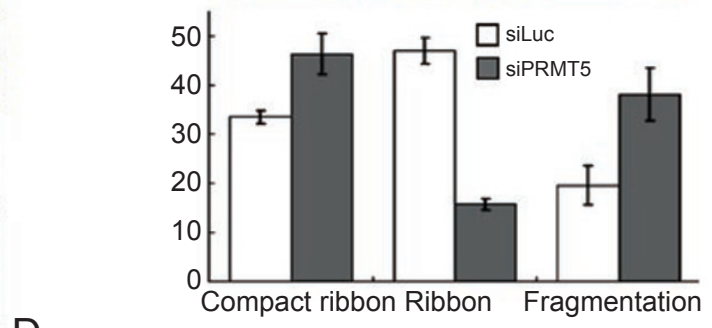

$\mathrm{D}$

$E$

F

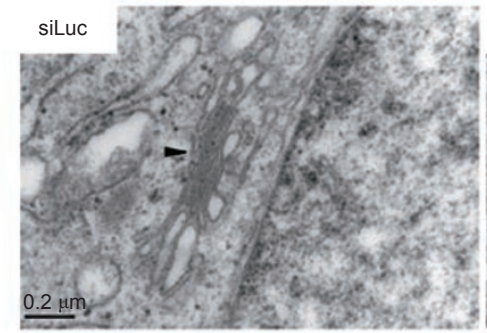

SiPRMT5
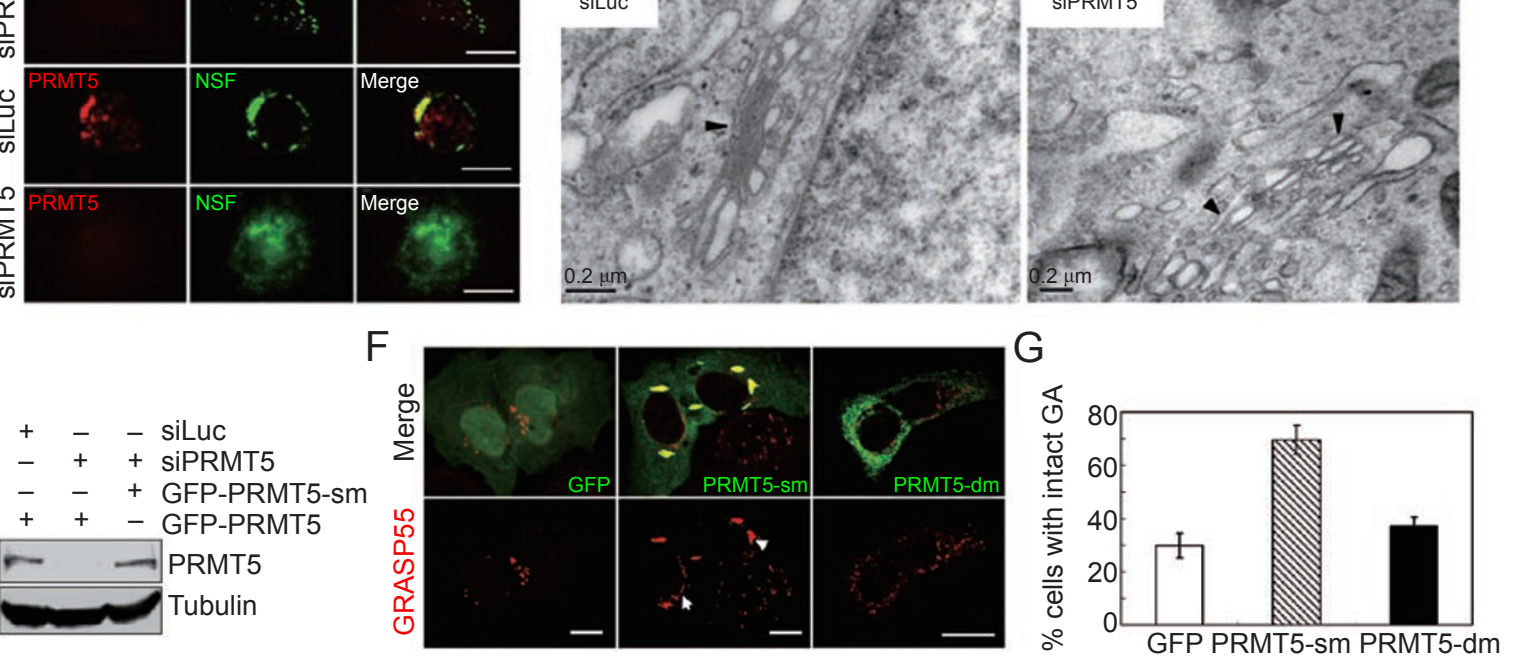

Figure 3 Disruption of Golgi structure through shRNA-mediated PRMT5 depletion. (A) Extracts of U2OS cells 72 h after transfection of siLuc or siPRMT5 vectors were immunoblotted with antibodies against PRMT5 and actin. (B) U2OS cells transfected with siLuc or siPRMT5 vectors were fixed $72 \mathrm{~h}$ after transfection, immunostained with antibodies against PRMT5 and GA markers GM130, p230, and NSF, and analyzed by confocal microscopy. Scale bars, $10 \mu \mathrm{m}$. (C) U2OS cells transfected siLuc-GFP or siPRMT5-GFP vectors were fixed $72 \mathrm{~h}$ after transfection, immunostained with antibodies against GA markers GRASP55, p230, and golgin-84, and cell population with three different GA structures were scored from more then 200 GFP-positive cells for each sample. Error bars indicate SD resulted from three independent experiments. The result obtained from GRASP55-stained cells is shown. (D) Cells treated as B were fixed, embedded, and imaged by transmission electron microscopy; GA was indicated by arrowhead. Scale bars, $200 \mathrm{~nm}$. (E) U2OS cells were cotransfected with constructs as indicated, and cell lysates were immunoblotted with anti-GFP and anti- $\beta$-tubulin antibodies. (F) U2OS cells cotransfected with vectors of GFP, GFP-PRMT5-sm, and GFP-RMT5-dm along with siPRMT5 (DNA molar ratio, 1:6) were fixed $48 \mathrm{~h}$ after transfection and stained with anti-GRASP55 (red) antibody; GA ribbon (arrowhead) was rescued in GFP-PRMT5-sm-positive cell; scale bar, $10 \mu \mathrm{m}$. (G) Quantitation of rescued GA structure in F. 150-200 GFP-positive cells were scored for each sample and repeated three times. Error bars represent standard deviation.

To confirm the mass spectrometry results, we generated a series of GM130 mutants by substituting, either individually or in combination, arginines 6, 18 and 23 with lysines, in GST-GM130N73 (Figure 4C). In vitro methylation of these mutant substrates with purified Flag-PRMT5 showed that mutations in all three positions affected GM130 methylation, as shown by the signifi- cantly diminished methylation signals in these mutants. This result suggests that the N-terminal arginine cluster is likely a methylation target of the PRMT5 methyltransferase activity. The fact that the presence of all three arginines was required for maximum methylation may suggest a potential coordination among these sites. In addition, a single site mutation at R18 completely abolished 

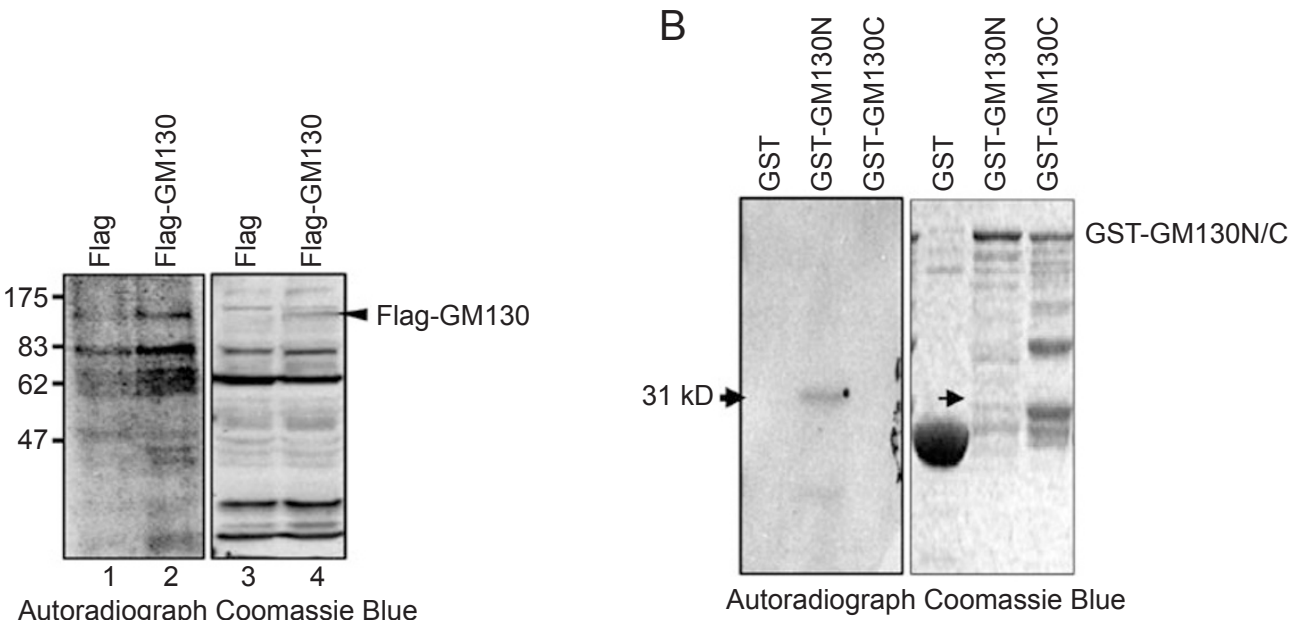

Autoradiograph Coomassie Blue
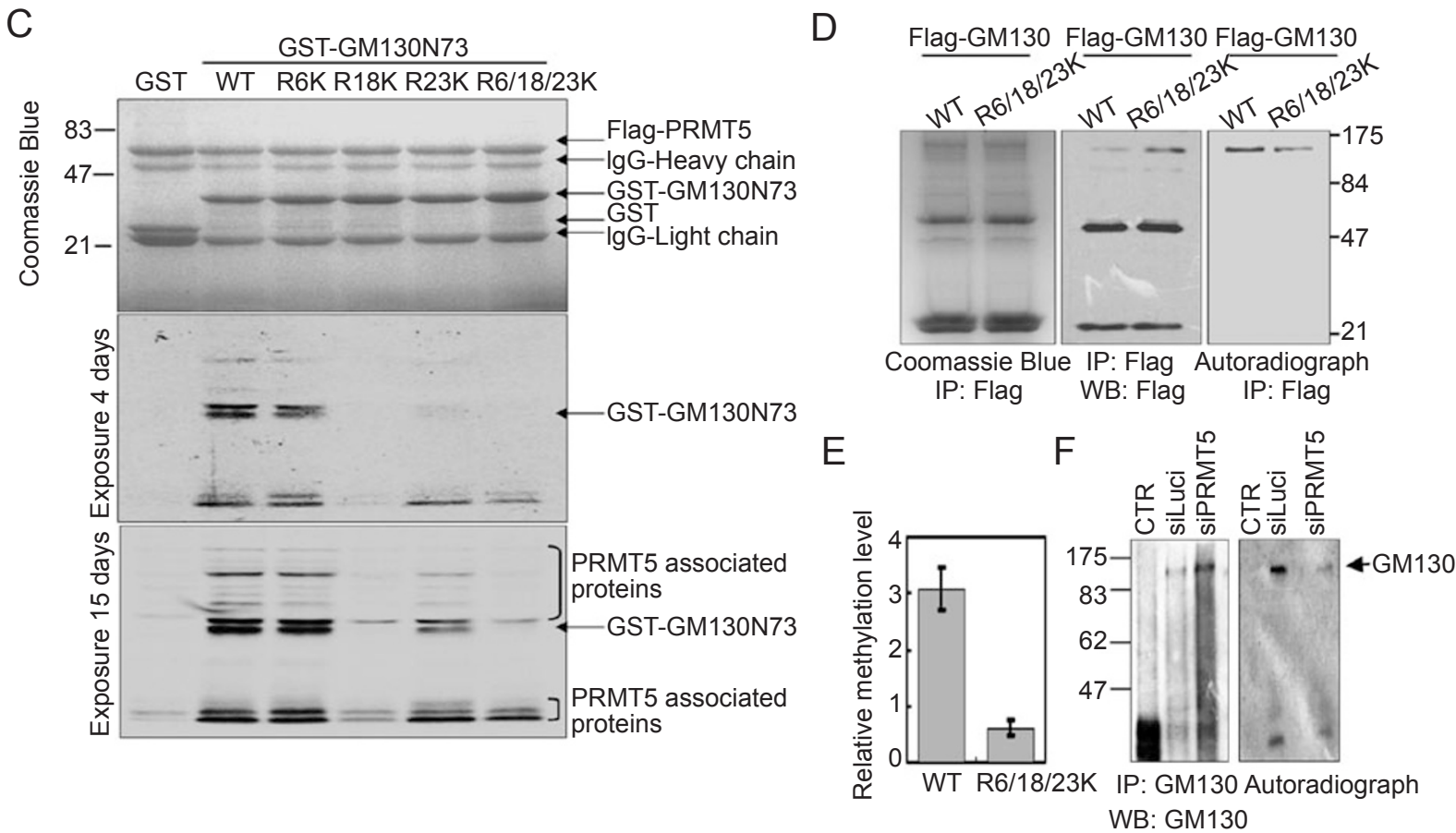

Figure 4 Identification of GM130 arginine methylation. (A) Autoradiograph of ${ }^{3} \mathrm{H}$-labeled Flag-GM130 (arrowhead) resulted from methylation assay: $20 \mu \mathrm{l}$ of Flag-PRMT5 pull down beads incubated with $20 \mu \mathrm{l}$ elution of either Flag-GM130 or Flag, which was transiently expressed in $\mathrm{CHO}$ cells. (B) Autoradiograph of ${ }^{3} \mathrm{H}$-labeled protein derived from in vitro methylation reaction with purified GST-PRMT5, and with GST $(10 \mu \mathrm{g})$, GST-GM130N $(5 \mu \mathrm{g})$, or GST-GM130C $(5 \mu \mathrm{g})$. Arrow indicates methylated GM130 N-terminus. (C) Methylation assay in vitro. Arginine residues in GM130N73 mutants were mutated to lysine (R6K, R18K, R23K). 2-5 $\mu \mathrm{g}$ of GST, GST-GM130N73, and GST-GM130N73 mutants were purified from E. coli, and incubated with $1 \mu \mathrm{g}$ of Flag-PRMT5 purified from HEK293T cells. Incorporation of ${ }^{3} \mathrm{H}$-labeled methyl groups (from the SAM donor) was monitored by autoradiography. (D) In vivo methylation of Flag-tagged GM130 and GM130 mutant transiently expressed in HEK293T cells in the presence of S-adenosyl-L- ${ }^{3} \mathrm{H}$-methyl]-methionine. GM130 and GM130 mutant proteins were affinity purified with beads coupled with Flag antibody. Elutes were subjected to SDS-PAGE and stained with Coomassie blue (left), western blotted with anti-FLAG antibodies (middle), or subjected to autoradiography (right). (E) Methylation levels in D were normalized to the amount of immunoprecipatated proteins. (F) In vivo methylation of GM130. GM130 were immunoprecipatated from HCT116-Lucif and HCT116 PRMT5-deleted cells in the presence of S-adenosyl-L-[ ${ }^{3} \mathrm{H}-$ methyl]-methionine for $3 \mathrm{~h}$. One-fifth eluate was subjected to western blotting with anti-GM130 antibodies (left) and four-fifth were subjected to autoradiography (right). 
Interestingly, the methylation levels of PRMT5-associated proteins other than GM130 were also increased by adding either immunoprecipatated Flag-GM130 or purified GST-GM130N73-WT (Figure 4A and 4C). Compared to the strong methylation signal in GSTGM130N73-WT, the methylation levels of PRMT5-associated proteins became weaker gradually by mutation of R6K, R23K, R18K, or R6/18/23K (Figure 4C), which corresponded to the methylation levels of the mutants themselves, suggesting that GM130 methylation might influence PRMT5 enzymatic activity.

To determine whether GM130 in fact contained methylated arginine residues in cells, Flag-GM130 was affinity purified from transiently transfected HEK293T cells and tryptic peptides were analyzed by mass spectrometry. A peptide with the observed molecular mass of 2166.02 Da was identified, which corresponded to residues 17-34 with two potential dimethylarginines and one phosphoserine (S25) residue (Supplementary information, Figure S6), consistent with the in vitro biochemical assay (Figure 4C) and previous studies on GM130 phosphorylation [6,
12]. In contrast to the in vitro methylation assay, neither monomethylarginine nor dimethylarginine corresponding to Arg6 (R6) was observed, and a peptide containing nonmethylated arginine at R6 was observed, suggesting that this residue may not be methylated in vivo (data not shown).

To further determine whether GM130 contains methylated arginines at the $\mathrm{N}$-terminal arginine residues in vivo, we transfected Flag-GM130 or Flag-GM130 ${ }^{\mathrm{R} 6 / 18 / 23 \mathrm{~K}}$ into HEK293 T cells, and labeled cell extracts with $\left[{ }^{3} \mathrm{H}\right.$-methyl]-SAM. When Flag-GM130 and Flag$\mathrm{GM} 130^{\mathrm{R} 6 / 18 / 23 \mathrm{~K}}$ proteins were immunoprecipitated from labeled cell extracts, we clearly detected ${ }^{3} \mathrm{H}$-methyl-FlagGM130, but observed a weaker signal with the FlagGM130 ${ }^{\text {R6/18/23K }}$ mutant (Figure 4D and 4E). To confirm whether GM130 methylation was catalyzed by PRMT5 in cells, we further examined the endogenous GM130 methylation levels in PRMT5-deleted cells. As shown in Figure 4F, GM130 was labeled with [ ${ }^{3} \mathrm{H}$-methyl]-SAM in cells transfected with control siRNA. In contrast, GM130 methylation levels decreased significantly in cells trans-

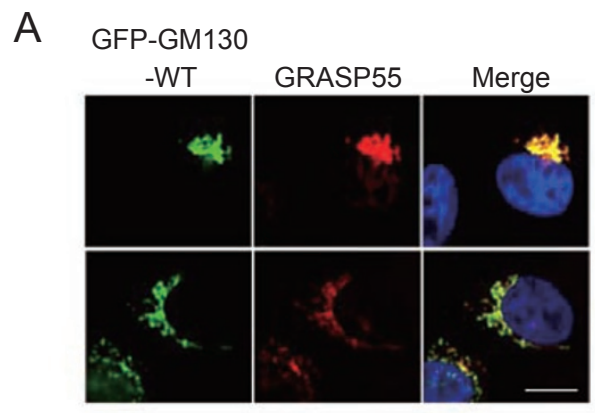

C

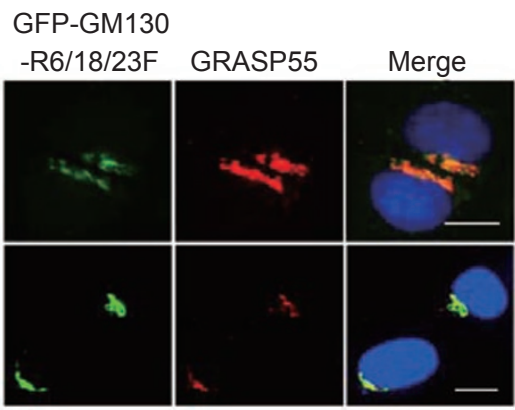

B

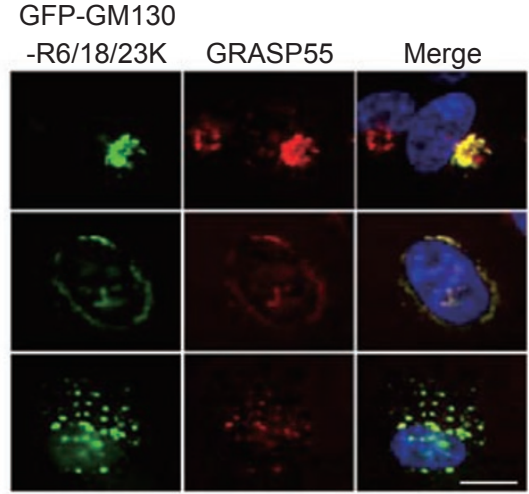

D

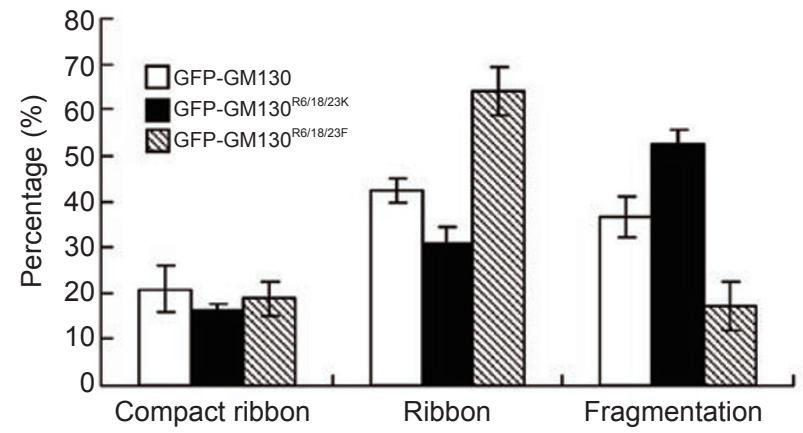

Figure 5 Functional analysis of GM130 arginine methylation. GFP-GM130 (A) or GFP-GM130 ${ }^{\text {R6/18/23K }}$ methylation mutant (B), and/or GFP-GM130 ${ }^{\mathrm{R} 6 / 18 / 23 \mathrm{~F}}$ constitutive methylation mutant (C) were expressed in U2OS cells, and after $72 \mathrm{~h}$ transfection, cells were fixed and stained with GRASP55 antibodies. (D) Cell populations with the three indicated GA morphologies were scored from more then 200 GFP-positive cells for each sample and error bars indicate SD from three independent experiments. Scale bars, $10 \mu \mathrm{m}$. 
fected with siPRMT5. These data, together with the methylation analysis in vitro, indicate that GM130 is indeed arginine methylated by PRMT5.

\section{GM130 arginine methylation influences GA structure}

To reveal the functional impact of GM130 methylation on GA structure, we generated a GFP-tagged GM130 $0^{\mathrm{R} 6 / 18 / 23 \mathrm{~K}}$ mutant and transiently expressed it in U2OS cells. Both GFP-GM130 and the GFP-GM130 ${ }_{\mathrm{R} 6 / 18 / 23 \mathrm{~K}}$ mutant were found to colocalize with the GA proteins GRASP55 (Figure 5A and 5B), p230, and golgin-84 (data not shown). However, compared to the normal GA structure observed in cells expressing wild-type GFPGM130, cells expressing the GFP-GM130 ${ }^{\mathrm{R} 6 / 18 / 23 \mathrm{~K}}$ mutant exhibited more fragmented and dispersed staining signals (Figure 5A, 5B, 5D).

We also generated a mutant GFP-GM130 $0^{\mathrm{R} 6 / 18 / 23 / \mathrm{F}}$ that mimics constitutive methylation through replacement of Arg6, Arg18, and Arg23 with Phe(F). The fluorescence of the GFP-GM130 $136 / 18 / 23 / \mathrm{F}$ mutant overlapped with GRASP55, indicating that it is correctly targeted to the GA (Figure 5C). Expression of the GFP-GM130 R6/18/23/F mutant significantly increased the number of cells with clear ribbon architecture (Figure 5D), further supporting the hypothesis that GM130 methylation affects GA ribbon formation. Thus, this provides a mechanistic basis for the PRMT5-dependent regulation of GA ribbon formation.

\section{Discussion}

In this work, we reveal the presence of PRMT5 complexes containing the Golgi-associated proteins GM130, NSF, and IQGAP1. Thus, an important physiological function of PRMT5 association with Golgi proteins may be to regulate various Golgi-dependent functions, including the structural organization of the organelle itself. PRMT5 localizes to the GA and loss of PRMT5 disrupts GA ribbon structure, suggesting that PRMT5 functions directly in GA architecture maintenance. This explains previous reports that PRMT5 dominantly localizes to cytoplasm in the somatic cells $[42,43]$ and plays a role in regulating pluripotency of germ line cells $[27,35,36]$ and in transformed B-lymphocyte growth and pathology $[32,44]$. Second, we identify a novel role for arginine methylation in regulation of Golgi structure, extending the role of PRMT5 and providing the first evidence of a functional role for methylation of Golgi proteins. This is consistent with the notion that arginine methylation exists in several Golgi proteins [17].

We propose that PRMT5 functions directly in maintenance of GA architecture through its arginine methy- lation of GM130, which is involved in GA stacking and ribbon assembly $[5,10,12]$. GM130 knockdown prevents lateral fusion between Golgi stacks resulting in the formation of ministacks [10]. Overexpression of the GFP-GM130 methylation mutant induced GA fragmentation (Figure 5B), a phenotype similar to GM130 depletion. Furthermore, the expression of a GM130 constitutive methylation mutant increased GA ribbon formation (Figure 5C and 5D). These results suggest that methylation of GM130 influences its role in linking Golgi membranes to the typical ribbon found in vertebrates.

Wild-type GM130 appears to influence PRMT5 enzymatic activity, but the GM130 methylation mutant could not (Figure 4A and 4C). In addition, the methyltransferase-dead mutant GFP-PRMT5-dm could not rescue the GA fragmentation induced by PRMT5 depletion (Figure 3E-3G), suggesting a plausible mechanism that overexpressed GFP-GM130 mutant might either inhibit the endogenous GA-localized PRMT5 activity, which results in the GA ribbon fragmentation, or impair the tethering complexes of GM130-p115-GRASP65 and GM130-p115-Giantin, which are critical to the GA structure [5-7, 10, 12, 45-49].

Golgi assembly and disassembly is a dynamic process coordinated in the cell cycle. Phosphorylation of GM130 and other Golgi-associated proteins in mitosis is thought to trigger Golgi disassembly. At present, it remains unknown whether or how PRMT5-mediated GM130 methylation contributes to the cell cycle regulation.

In summary, our results reveal a new function for arginine methylation in the cytoplasm, namely, the regulation of GA structural organization. We reveal that a major function of PRMT5 is in regulation of GA structure through methylation of GM130.

\section{Materials and Methods}

\section{DNA constructs and antibodies}

Human PRMT5 full-length cDNA (GeneBank accession number: BC025979) and GM130 (GeneBank accession number: BC069268) were purchased from Open Biosystems. PRMT5, p115, GM130, GM130N (1-500 aa), GM130N 473 (74-500 aa), GM130C (497-990 aa), and GM130N73 (1-73 aa) were subcloned into pEGFPC1 (Clontech), pGEX4T-1, and pCMV-Tag 2A/2B (Stratagene) in frame by PCR. The U6 promoter-driven shRNA expression vector pDsU6 and control shRNA (siLuc) were previously described [41]. Four short hairpin-based shRNA silencing vectors were generated, which were specific for target PRMT5 mRNA codon sequence. The target sites in the PRMT5 coding region were 288-308, 626-646, 848-868, and 1 221-1 241 (+1 being the A residue in the initiating codon) and were verified in the human genome sequence database (NCBI) as unique sequences. Two high-efficiency vectors pDs288 and pDs626 were named as siPRMT5-1 and siPRMT5-2, respectively. siPRMT5 indicates 
siPRMT5-1 if not specified. PRMT5 shRNA-resistant mutant (pGFP-PRMT5-sm) construct was prepared by introducing two silent mutations within the siPRMT5-targeting sequence using sitedirected mutagenesis with the sequence 5'-TGG AGA AGA TTC GCC GGA ATT CCG AGG C-3'. PRMT5 methyltransferase-dead mutant (PRMT5-md, G367A/R368A) and GM130N73 serial mutants were generated with site-directed mutagenesis. All constructs were verified by double-stranded DNA sequencing. The polyclonal antibody against PRMT5 was purchased from Upstate. The monoclonal antibodies against $\beta$-actin, $\alpha$-tubulin, GST, Flag, and ManII were products of Sigma. Monoclonal antibody against GFP was purchased from Clontech. Anti-GM130, anti-golgin-84, anti-NSF, anti-Bip, anti-EEA1, anti-Giantin, anti-Bcl2, anti-Annexin II, and anti-Calnexin monoclonal antibodies were from BD Transduction Laboratories. The polyclonal antibodies against GRASP55 and p115 were made by Animal Center in Institute of Genetics and Developmental Biology.

\section{PRMT5 complex purification and reciprocal immunopre- cipitation}

For reciprocal immunoprecipitation, $500 \mu \mathrm{g}$ of cell extract protein was incubated overnight with $2 \mu \mathrm{g}$ of anti-GM130, antiPRMT5, anti-Flag antibodies or IgG and $10 \mu \mathrm{l}$ of Protein A/G agarose beads (OncogeneScience) at $4{ }^{\circ} \mathrm{C}$. After extensive washing with buffer $(50 \mathrm{mM}$ Tris- $\mathrm{HCl}, 150 \mathrm{mM} \mathrm{NaCl}, 0.5 \%$ Triton X-100 ( $\mathrm{pH} 7.4)$ ), all associated proteins were eluted and subjected to western blot.

Cell culture, transfection, western blot, and immunofluorescence microscopy

HCT116, HEK293 T, MCF7, CHO, and U2OS cells were maintained at $37{ }^{\circ} \mathrm{C}$ and $5 \% \mathrm{CO}_{2}$ in Dulbecco's modified Eagle's medium (Gibco) plus 10\% fetal bovine serum (Gibco) and 1\% penicillin-streptomycin (Gibco). Cells were transfected using Lipofectamine 2000 (Invitrogen) following manufacturer's recommendations. HCT116 or U2OS cells transfected with siPRMT5 construct or the control siLuc were grown in the presence of G-418 (Gibco) for 48 or $72 \mathrm{~h}$ to enrich for transfected cells, as described previously [41]. These transfected cells were harvested for western blot or fixed for electron microscopy assay. For immunofluorescence staining, cells grown on coverslips were either fixed to stain with indicated antibodies or cotransfected with the siPRMT5 vector and GFP or GFP-tagged PRMT5 vectors (6:1 molar ratio), and then fixed to stain with Golgi marker antibodies as described [41]. The stained cells were visualized under a Zeiss or Nikon microscope. For electron microscopy, cells were fixed with $2 \%$ glutaraldehyde in $0.1 \mathrm{M}$ sodium cacodylate buffer $(\mathrm{pH} 7.2)$ for $1 \mathrm{~h}$, postfixed with $1 \%$ osmium tetroxide for $1 \mathrm{~h}$, dehydrated in alcohols, and embedded in Epon. Sections were cut on ultramicrotome and analyzed with a JEM-1230 transmission electron microscope (JEOL, Tokyo, Japan).

\section{Protein purification and methylation analysis}

GST and GST-fused proteins expression, purification, and methylation assays were carried out as previously described [30]. For analysis of full-length GM130 methylation, $\mathrm{CHO}$ cells were transfected with pCMV-TAG-2B-GM130 and harvested $24 \mathrm{~h}$ after transfection with lysis buffer $(50 \mathrm{mM}$ Tris- $\mathrm{HCl}, 150 \mathrm{mM} \mathrm{NaCl}, 1$ mM EDTA, and $0.5 \%$ Triton X-100 with protease inhibitor cock- tail (Roche) (pH 7.4)); Flag-GM130 was purified by anti-Flag M2 affinity beads (Sigma), and was eluted with two volumes of elution buffer $(50 \mathrm{mM}$ Glycine- $\mathrm{HCl}(\mathrm{pH} 2.7))$ and then neutralized by neutralization buffer $(0.5 \mathrm{M}$ Tris- $\mathrm{HCl}, 1.5 \mathrm{M} \mathrm{NaCl}, \mathrm{pH} 8.0)$. About $1 \mu \mathrm{g}$ of Flag-PRMT5-binding beads purified from $\mathrm{CHO}$ cells were mixed with $5 \mu \mathrm{l}$ of ${ }^{3} \mathrm{H}-\mathrm{SAM}$ and $20 \mu \mathrm{l}$ of Flag-GM130 elutes, and the mixture was incubated at $30{ }^{\circ} \mathrm{C}$ for $3 \mathrm{~h}$ and then stopped by adding SDS sample buffer, boiled for $5 \mathrm{~min}$ at $100{ }^{\circ} \mathrm{C}$ and subjected to SDS-PAGE. The mixture was split into two parts: the gel with 9/10 mixture was stained by Coomassie Blue, destained, incubated with enhancer buffer for $1 \mathrm{~h}$, dried, and exposed on $\mathrm{X}$ film for 10-20 days; the gel with 1/10 mixture was transferred to Nylon membrane for western blotting with anti-Flag antibody. For in vivo labeling, at $24 \mathrm{~h}$ after being transfected with Flag-GM130or Flag-GM130 $0^{\mathrm{R} 6 / 18 / 23 \mathrm{~K}}$-expressing vectors, HEK239T cells were fed with ${ }^{3} \mathrm{H}-\mathrm{SAM}(0.55 \mu \mathrm{Ci} / \mathrm{ml})$ for $1 \mathrm{~h}$ at $37^{\circ} \mathrm{C}$ and then washed with ice-cold $1 \times$ PBS $(\mathrm{pH} 8.0)$ three times; clarified whole cell lysate protein $(2 \mathrm{mg}$ ) was used for purification of Flag-GM130 or Flag-GM130 $0^{\mathrm{R} 6 / 18 / 23 \mathrm{~K}}$. Four-fifth of Flag immunoprecipitates were subjected to autoradiography and one-fifth was used for western blotting by anti-Flag antibody.

\section{Rat liver Golgi membrane isolation}

Isolation of Rat liver Golgi membranes was conducted as previously described [49-50]. Six rat livers were homogenized in $\mathrm{K} /$ $\mathrm{S}$ buffer $\left(0.1 \mathrm{M} \mathrm{K}_{2} \mathrm{H}_{2} \mathrm{PO}_{4}\right.$ and $5 \mathrm{mM} \mathrm{MgCl}$ ) with $0.5 \mathrm{M}$ sucrose. $4 \mathrm{ml}$ of homogenate was laid on top of $6.5 \mathrm{ml}$ of $0.86 \mathrm{M} \mathrm{K} / \mathrm{S}$ buffer in SW40 tubes, and overlaid with $2.5 \mathrm{ml}$ of $0.25 \mathrm{M}$ sucrose $\mathrm{K} /$ $\mathrm{S}$ buffer. After centrifugation at 29000 r.p.m. for $60 \mathrm{~min}$ at $4{ }^{\circ} \mathrm{C}$, the interface of $0.5 \mathrm{M}$ sucrose and $0.86 \mathrm{M}$ sucrose $\mathrm{K} / \mathrm{S}$ buffer was collected and adjusted to $0.25 \mathrm{M}$ sucrose $\mathrm{K} / \mathrm{S}$. This was laid on top of $1.5 \mathrm{ml}$ of $0.35 \mathrm{M} \mathrm{K} / \mathrm{S}$ buffer, which was laid on top of 0.5 $\mathrm{ml} 1.2 \mathrm{M} \mathrm{K} / \mathrm{S}$ buffer in SW40 tubes. After centrifugation at 6000 r.p.m. for $20 \mathrm{~min}$ at $4{ }^{\circ} \mathrm{C}$, the Golgi membranes at the $0.35 \mathrm{M} / 1.2$ $\mathrm{M}$ sucrose interface were collected and snap frozen in liquid nitrogen for storage at $-80^{\circ} \mathrm{C}$. The Golgi membranes were subjected to western blot.

\section{Large-scale PRMT5 immunoprecipitaiton}

Hela cells were harvested and washed three times by PBS. The cell pellet was gently resuspended with five volumes of hypotonic buffer (10 mM HEPES-KOH (pH 8.0), $10 \mathrm{mM} \mathrm{KCl,} 1.5 \mathrm{mM}$ $\mathrm{MgCl}_{2}, 1 \mathrm{mM}$ dithiothreitol (DTT), and $0.2 \mathrm{mM}$ phenylemethylsulfonyl fluoride (PMSF)) for $10 \mathrm{~min}$ on ice. Then the pellets were added to two volumes of hypotonic buffer and homogenized with 15 strokes using pestle B in a Dounce glass homogenizer (Wheaton, Millville, NJ, USA). The lysate was centrifuged at $20000 \times g$ for $30 \mathrm{~min}$ at $4{ }^{\circ} \mathrm{C}$. The supernatant was mixed with 0.11 volume of high-salt buffer (20 mM HEPES-KOH (pH 8.0), 20\% glycerol, 1.2 $\mathrm{M} \mathrm{KCl}, 1.5 \mathrm{mM} \mathrm{MgCl}, 0.2 \mathrm{mM}$ EDTA, $1 \mathrm{mM}$ DTT, and $0.2 \mathrm{mM}$ $\mathrm{PMSF}$ ) and centrifuged at $100000 \times g$ for $1 \mathrm{~h}$ at $4{ }^{\circ} \mathrm{C}$. The supernatant was dialyzed against dialysis buffer for $2 \mathrm{~h}$ at $4{ }^{\circ} \mathrm{C}$ and then centrifuged at $20000 \times g$ for $30 \mathrm{~min}$ at $4{ }^{\circ} \mathrm{C}$. The supernatant $(15$ $\mathrm{mg}$ ) was mixed with $20 \mu \mathrm{g}$ PRMT5 antibody and rotated for $4 \mathrm{~h}$ at $4{ }^{\circ} \mathrm{C}$ and then mixed with $50 \mu$ l of protein A-Sepharose beads $(50 \%$ slurry) for $1 \mathrm{~h}$ at $4{ }^{\circ} \mathrm{C}$. The immunoprecipitates were washed three times with the buffer (20 mM Tris- $\mathrm{HCl}$ (pH 8.0), $100 \mathrm{mM} \mathrm{NaCl}, 1$ mM EDTA, and $0.5 \%$ Nonidet P-40). The beads were boiled with loading buffer and subjected to SDS-PAGE. The Coomassie blue- 
stained protein bands were excised from the gel and subjected to in-gel digestion and mass spectrometry analysis.

\section{Acknowledgments}

The authors thank Dr Martin Lowe at the University of Manchester for critical comments on the manuscript. We also thank Dr Zhukuang Cheng at IGDB, confocal microscope facility at the Institute of Biophysics and IGDB and EM facility at the Institute of Botany for providing assistance to perform immunofluorescence and electron microscopy. We acknowledge financial support from the National Basic Research Program of China (2005CB22400), National High Technology Development Program (2006AA02Z140), Chinese Academy of Sciences (KSCX1YW-R-59), and National Natural Sciences Foundation of China (30670479).

\section{References}

1 Shorter J, Warren G. Golgi architecture and inheritance. Annu Rev Cell Dev Biol 2002; 18:379-420.

2 Colanzi A, Suetterlin C, Malhotra V. Cell-cycle-specific Golgi fragmentation: how and why? Curr Opin Cell Biol 2003; 15:462-467.

3 Barr FA, Short B. Golgins in the structure and dynamics of the Golgi apparatus. Curr Opin Cell Biol 2003; 15:405-413.

4 Ramirez IB, Lowe M. Golgins and GRASPs: holding the Golgi together. Semin Cell Dev Biol 2009; 20:770-779.

5 Nakamura N, Rabouille C, Watson R, et al. Characterization of a cis-Golgi matrix protein, GM130. J Cell Biol 1995; 131:1715-1726.

6 Nakamura N, Lowe M, Levine TP, Rabouille C, Warren G. The vesicle docking protein p115 binds GM130, a cis-Golgi matrix protein, in a mitotically regulated manner. Cell 1997; 89:445-455.

7 Marra P, Maffucci T, Daniele T, et al. The GM130 and GRASP65 Golgi proteins cycle through and define a subdomain of the intermediate compartment. Nat Cell Biol 2001; 3:1101-1113.

8 Sonnichsen B, Lowe M, Levine T, et al. A role for giantin in docking COPI vesicles to Golgi membranes. J Cell Biol 1998; 140:1013-1021.

9 Beard M, Satoh A, Shorter J, Warren G. A cryptic Rab1binding site in the p115 tethering protein. J Biol Chem 2005; 280:25840-25848

10 Puthenveedu MA, Bachert C, Puri S, Lanni F, Linstedt AD. GM130 and GRASP65-dependent lateral cisternal fusion allows uniform Golgi-enzyme distribution. Nat Cell Biol 2006; 8:238-248.

11 Vasile E, Perez T, Nakamura N, Krieger M. Structural integrity of the Golgi is temperature sensitive in conditional-lethal mutants with no detectable GM130. Traffic 2003; 4:254-272.

12 Lowe M, Gonatas NK, Warren G. The mitotic phosphorylation cycle of the cis-Golgi matrix protein GM130. J Cell Biol 2000; 149:341-356.

13 Preisinger C, Korner R, Wind M, et al. Plk1 docking to GRASP65 phosphorylated by Cdk1 suggests a mechanism for Golgi checkpoint signalling. EMBO J 2005; 24:753-765.

14 Diao A, Rahman D, Pappin DJ, Lucocq J, Lowe M. The coiled-coil membrane protein golgin-84 is a novel rab effector required for Golgi ribbon formation. J Cell Biol 2003; 160:201-212.

15 Behnia R, Panic B, Whyte JR, Munro S. Targeting of the Arflike GTPase Arl3p to the Golgi requires N-terminal acetylation and the membrane protein Sys1p. Nat Cell Biol 2004; 6:405-413.

16 Wang Y, Satoh A, Warren G, Meyer HH. VCIP135 acts as a deubiquitinating enzyme during p97-p47-mediated reassembly of mitotic Golgi fragments. J Cell Biol 2004; 164:973978.

17 Wu CC, MacCoss MJ, Mardones G, et al. Organellar proteomics reveals Golgi arginine dimethylation. Mol Biol Cell 2004; 15:2907-2919.

18 Pollack BP, Kotenko SV, He W, et al. The human homologue of the yeast proteins Skb1 and Hsl7p interacts with Jak kinases and contains protein methyltransferase activity. $J$ Biol Chem 1999; 274:31531-31542.

19 Gilbreth M, Yang P, Bartholomeusz G, et al. Negative regulation of mitosis in fission yeast by the shk1 interacting protein skb1 and its human homolog, Skb1Hs. Proc Natl Acad Sci USA 1998; 95:14781-14786.

20 Chari A, Golas MM, Klingenhager M, et al. An assembly chaperone collaborates with the SMN complex to generate spliceosomal SnRNPs. Cell 2008; 135:497-509.

21 Kwak YT, Guo J, Prajapati S, et al. Methylation of SPT5 regulates its interaction with RNA polymerase II and transcriptional elongation properties. Mol Cell 2003; 11:1055-1066.

22 Wang X, Zhang Y, Ma Q, et al. SKB1-mediated symmetric dimethylation of histone H4R3 controls flowering time in Arabidopsis. EMBO J 2007; 26:1934-1941.

23 Zhao Q, Rank G, Tan YT, et al. PRMT5-mediated methylation of histone H4R3 recruits DNMT3A, coupling histone and DNA methylation in gene silencing. Nat Struct Mol Biol 2009; 16:304-311.

24 Bedford MT, Richard S. Arginine methylation an emerging regulator of protein function. Mol Cell 2005; 18:263-272.

25 Pal S, Vishwanath SN, Erdjument-Bromage H, Tempst P, Sif S. Human SWI/SNF-associated PRMT5 methylates histone $\mathrm{H} 3$ arginine 8 and negatively regulates expression of ST7 and NM23 tumor suppressor genes. Mol Cell Biol 2004; 24:96309645.

26 Jansson M, Durant ST, Cho EC, et al. Arginine methylation regulates the p53 response. Nat Cell Biol 2008; 10:14311439.

27 Ancelin K, Lange UC, Hajkova P, et al. Blimp1 associates with Prmt5 and directs histone arginine methylation in mouse germ cells. Nat Cell Biol 2006; 8:623-630.

28 Gonsalvez GB, Rajendra TK, Tian L, Matera AG. The Smprotein methyltransferase, dart5, is essential for germ-cell specification and maintenance. Curr Biol 2006; 16:10771089.

29 Dacwag CS, Bedford MT, Sif S, Imbalzano AN. Distinct protein arginine methyltransferases promote ATP-dependent chromatin remodeling function at different stages of skeletal muscle differentiation. Mol Cell Biol 2009; 29:1909-1921.

30 Bao S, Qyang Y, Yang P, et al. The highly conserved protein methyltransferase, Skb1, is a mediator of hyperosmotic stress response in the fission yeast Schizosaccharomyces pombe. $J$ 
Biol Chem 2001; 276:14549-14552.

31 Rho J, Choi S, Seong YR, et al. Prmt5, which forms distinct homo-oligomers, is a member of the protein-arginine methyltransferase family. J Biol Chem 2001; 276:11393-11401.

32 Pal S, Baiocchi RA, Byrd JC, et al. Low levels of miR-92b/96 induce PRMT5 translation and H3R8/H4R3 methylation in mantle cell lymphoma. EMBO J 2007; 26:3558-3569.

33 Hou Z, Peng H, Ayyanathan K, et al. The LIM protein AJU$\mathrm{BA}$ recruits protein arginine methyltransferase 5 to mediate SNAIL-dependent transcriptional repression. Mol Cell Biol 2008; 28:3198-3207.

34 Eckert D, Biermann K, Nettersheim D, et al. Expression of BLIMP1/PRMT5 and concurrent histone $\mathrm{H} 2 \mathrm{~A} / \mathrm{H} 4$ arginine 3 dimethylation in fetal germ cells, CIS/IGCNU and germ cell tumors. BMC Dev Biol 2008; 8:106.

35 Durcova-Hills G, Tang F, Doody G, Tooze R, Surani MA. Reprogramming primordial germ cells into pluripotent stem cells. PLoS One 2008; 3:e3531.

36 Eguizabal C, Shovlin TC, Durcova-Hills G, Surani A, McLaren A. Generation of primordial germ cells from pluripotent stem cells. Differentiation 2009; 78:116-123.

37 Meister G, Fischer U. Assisted RNP assembly: SMN and PRMT5 complexes cooperate in the formation of spliceosomal UsnRNPs. EMBO J 2002; 21:5853-5863.

38 Malhotra V, Orci L, Glick BS, Block MR, Rothman JE. Role of an N-ethylmaleimide-sensitive transport component in promoting fusion of transport vesicles with cisternae of the Golgi stack. Cell 1988; 54:221-227.

39 Izumi G, Sakisaka T, Baba T, et al. Endocytosis of E-cadherin regulated by Rac and $\mathrm{Cdc} 42$ small $\mathrm{G}$ proteins through IQGAP1 and actin filaments. $J$ Cell Biol 2004; 166:237-248.

40 Preisinger C, Short B, De Corte V, et al. YSK1 is activated by the Golgi matrix protein GM130 and plays a role in cell migration through its substrate 14-3-3zeta. J Cell Biol 2004; 164:1009-1020.
41 Bao S, Lu T, Wang X, et al. Disruption of the Rad9/Rad1/ Hus1 (9-1-1) complex leads to checkpoint signaling and replication defects. Oncogene 2004; 23:5586-5593.

42 Lee JH, Cook JR, Yang ZH, et al. PRMT7, a new protein arginine methyltransferase that synthesizes symmetric dimethylarginine. J Biol Chem 2005; 280:3656-3664.

43 Lee J, Sayegh J, Daniel J, Clarke S, Bedford MT. PRMT8, a new membrane-bound tissue-specific member of the protein arginine methyltransferase family. J Biol Chem 2005; 280:32890-32896.

44 Wang L, Pal S, Sif S. Protein arginine methyltransferase 5 suppresses the transcription of the RB family of tumor suppressors in leukemia and lymphoma cells. Mol Cell Biol 2008; 28:6262-6277.

45 Barr FA, Puype M, Vandekerckhove J, Warren G. GRASP65, a protein involved in the stacking of Golgi cisternae. Cell 1997; 91:253-262.

46 Lowe M, Rabouille C, Nakamura N, et al. Cdc2 kinase directly phosphorylates the cis-Golgi matrix protein GM130 and is required for Golgi fragmentation in mitosis. Cell 1998; 94:783-793.

47 Shorter J, Warren G. A role for the vesicle tethering protein, p115, in the post-mitotic stacking of reassembling Golgi cisternae in a cell-free system. J Cell Biol 1999; 146:57-70.

48 Alvarez C, Garcia-Mata R, Hauri HP, Sztul E. The p115-interactive proteins GM130 and giantin participate in endoplasmic reticulum-Golgi traffic. J Biol Chem 2001; 276:2693-2700.

49 Diao A, Frost L, Morohashi Y, Lowe M. Coordination of golgin tethering and SNARE assembly: GM130 binds syntaxin 5 in a p115-regulated manner. J Biol Chem 2008; 283:69576967.

50 Slusarewicz P, Nilsson T, Hui N, Watson R, Warren G. Isolation of a matrix that binds medial Golgi enzymes. J Cell Biol 1994; 124:405-413.

(Supplementary information is linked to the online version of the paper on Cell Research website.) 